I Universidade Federal do Rio de Janeiro (UFRJ), Departamento

de Antropologia Cultural, Rio de Janeiro, RJ, Brasil

rabossi@rocketmail.com

https://orcid.org/o0oo-0003-1227-5258

Fernando Rabossi'

\title{
LOS CAMINOS DE LA INFORMALIDAD
}

Durante mi trabajo de campo en Ciudad del Este (Paraguay) con diversos trabajadores envueltos en la actividad comercial, la categoría "informal" fue utilizada por algunos de ellos para autodefinirse. Sin embargo, qué significaba eso no era evidente. Para algunos, lo fundamental era el trabajo en relación de no-dependencia. ${ }^{\text {I }}$ Para otros, lo definía el no-pago de impuestos. ${ }^{2}$ Cada una de estas formas de concebir la informalidad sugería otras cuestiones vinculadas a actores, relaciones y contenidos particulares. Algunos elementos que surgieron bajo la figura de informalidad en el campo y en la literatura me ayudaron en el desarrollo de lo que después sería mi tesis de doctorado (Rabossi, 2004). No obstante, la informalidad no fue clave para su construcción ni tampoco uno de los ejes de discusión. Sentía que la discusión sobre informalidad en ciencias sociales estaba pautada por una serie de presupuestos de los cuales quería desprenderme para poder presentar una discusión sobre el material etnográfico sin visiones normativas y dicotómicas. Aun así, la informalidad estaba en la calle, siendo utilizada para definir y autodefinirse.

El presente trabajo aborda algunas de las discusiones que fueron moldeando el concepto de informalidad como un primer paso para comprender su difusión y utilización; elementos que nos permiten comprender cómo actores en lugares tan distantes como Nairobi, Nueva Delhi o Ciudad del Este se autoidentifican como "informales". Para eso, presentaré los trabajos donde éste fue inicialmente formulado, su incorporación en la Organización Internacional del 
Trabajo, y la historia institucional particular que lo transforma y amplifica. Recuperar algunos aspectos de la historia de la noción de informalidad permite retomar las discusiones sobre su desarrollo desde un nuevo ángulo y reflexionar sobre el gobierno de la economía, abriendo nuevos caminos de indagación histórica sobre las formas de modernización en la segunda mitad del siglo XX y las maneras de concebirlas. ${ }^{3}$

Si bien el punto de partida de este trabajo es etnográfico, el interés teórico es comprender la relación entre ideas económicas - aquellas producidas por especialistas - e ideas y prácticas ordinarias (Motta et al., 20I4: 50). Confluencia entre una sociología de las ideas, los estudios sociales de la ciencia y de la economía y las etnografías económicas, el esfuerzo aquí emprendido trata de

describir la construcción social de las teorías económicas y los complejos mecanismos que les otorgan legitimidad social y permiten que se diseminen más allá del círculo de especialistas [y] comprender los procesos mediante los cuales estas teorías convergen con otras dimensiones de la cultura y otras formas de conceptualizar las relaciones entre las personas y la vida social 4 (Neiburg, 2006: $630) .5$

Los caminos del concepto de informalidad - que, como veremos, empiezan con las "oportunidades informales" de empleo para desembocar en el "sector informal" - transcienden el campo de la economía y colocan en relación etnografías, estudios de caso, antropólogos, economistas y organismos internacionales. En este trabajo privilegio dos actores en particular: Keith Hart y Paul Bangasser. El primero, antropólogo, escribiendo desde su trabajo de campo en Ghana; el segundo, miembro de la Organización Internacional del Trabajo, testigo privilegiado de la trayectoria institucional del concepto en dicha organización. A partir de ellos, reconstituiremos una historia muchas veces contada, pero que puede ganar nuevos matices desde las posiciones singulares elegidas para contarla.

\section{KEITH HART Y LA FORMULACIÓN DE LA INFORMALIDAD}

Como toda historia conceptual, la de la informalidad tiene sus detalles poco conocidos, al menos para muchos antropólogos, porque muchos economistas - especialmente aquellos dedicados al desarrollo - conocen la disciplina y la persona asociada al surgimiento del concepto: el antropólogo Keith Hart, cuya etnografía en Ghana sirvió como plataforma de despegue del mismo.

Publicado en $1973,{ }^{6}$ el trabajo señalado como el primero en desarrollar la idea de informalidad, fue presentado por Keith Hart en septiembre de I97 I, en una conferencia sobre desempleo urbano en África. El mismo se desprende de la investigación del autor sobre un grupo del norte de Ghana, los Frafra, algunos de los cuales habían migrado a las ciudades del sur del país. El trabajo describe y analiza las estrategias de generación de ingresos de los inmigrantes en Accra 
- a quienes Hart denomina subproletariado urbano -, especialmente en el distrito de Nima, donde realizó parte importante de su trabajo de campo. Como los salarios no alcanzaban, las personas recurrían a diversas alternativas como crédito de familiares y de amigos, duplicación del trabajo asalariado, y diversos medios "informales" para incrementar los ingresos. Es precisamente este último mundo de actividades económicas, fuera de la "fuerza de trabajo organizada", que constituye el centro del artículo.

Para Hart, la distinción entre oportunidades formales e informales de ingreso está basada en la diferencia entre trabajo asalariado y autoempleo, siendo la variable principal de diferenciación el grado de racionalización del trabajo (Hart, I973: I47). Esa característica inscribe diferencialmente aquellas actividades que son enumerables en los relevamientos - constituyendo el "sector moderno" - y aquellas que escapan a la enumeración - lo que era descrito por otros autores como sector de baja productividad, ejército de reserva de subempleados y desempleados, o sector urbano tradicional. Estás últimas son definiciones que, según el autor, llevaban implícitas una descripción que de hecho debía ser demostrada.

Hart desarrolla una tipología que le permite presentar la amplitud de oportunidades de ingresos informales, distinguiendo entre actividades legítimas e ilegítimas; una distinción derivada de la ley, pero vinculada de forma genérica a lo que un "respetable ciudadano" piensa que es legítimo. La variedad de actividades de producción y servicios, distribución y transferencias privadas que aparecen a la luz de ese ejercicio tipológico, le permite a Hart explorar las causas y las dinámicas de esa diversidad: la distribución desigual de las oportunidades para los diferentes grupos étnicos, la diferente exposición a las reglas coloniales y a la educación occidental, los patrones restrictos de circulación de la información, y la conformación de nichos étnicos.

Después de presentar descriptivamente estos aspectos y de detenerse en el estudio de un caso, 7 Hart señala tres direcciones en las cuales deberían ser reorientados los trabajos. En primer lugar, debemos reformular las preguntas. Por ejemplo, no preguntarnos “¿cómo podemos hacer para crear empleo para los desempleados?” sino, “¿queremos colocar el énfasis en la dirección del empleo formal porque es bueno en sí?, ¿por qué queremos reducir las actividades socialmente desaprobadas? o ¿por qué queremos acabar con la ineficiencia de esos trabajos?" (Hart, I973: I57). En segundo lugar, debemos cuestionar las suposiciones que hasta entonces guiaron las propuestas de generación de empleo y de desarrollo en sentido más general. Por ejemplo, es absurdo pensar empírica y teóricamente en un proceso de transición del desempleo y subempleo a una situación de pleno empleo a través de la mera generación de empleo en la fuerza de trabajo organizada (Hart, I973: I58). En tercer lugar, debemos pensar abordajes que permitan dar cuenta de las estructuras de ingresos formales e informales en forma conjunta. ${ }^{8}$ 
Hart concluye su artículo llamando la atención sobre dos cuestiones. Accra no es única en las características descritas a lo largo del trabajo, por lo que se impone la necesidad de un abordaje comparativo de las economías urbanas que tome en consideración las estructuras formales e informales. A su vez, esto debe reconfigurar la agenda con la que se piensa el desarrollo. En sus palabras, "Llegó la hora para que el lenguaje y el abordaje de la economía del desarrollo tome esto en cuenta" (Hart, I973: I62). Vemos así que el interés de Hart era establecer un diálogo con los economistas del desarrollo para introducir un universo de actividades que generalmente eran desconsideradas o consideradas marginales al proceso económico. ${ }^{9}$

Tal como el propio autor lo formulara algunos años más tarde,

[L]a idea de una 'economía informal' surgió del deseo de comunicarme con los economistas del desarrollo a inicios de los años I970, desde la perspectiva de mi investigación etnográfica en un barrio de una ciudad de África Occidental. [...] Mi objetivo era mostrar que las actividades económicas autoorganizadas, emergiendo de la vida cotidiana de las personas, por más irregulares e inadecuadas que pudieran parecer, deberían modificar las percepciones de la economía presente en el discurso del desarrollo (Hart, 2006: I).

Sin embargo, el debate en el cual el concepto se inscribe se revela a la luz de otra vertiente donde el mismo se desarrolla: la Organización Internacional del Trabajo. En ella me detendré seguidamente.

\section{LA OIT Y LA POPULARIZACIÓN DEL CONCEPTO ${ }^{10}$}

Si hay una institución vinculada al concepto de sector informal, esa es la Organización Internacional del Trabajo (OIT). ${ }^{\text {II }}$ Para algunos, es considerada tanto la partera, como su principal hogar institucional. Para comprender esa relación es necesario trazar algunos desarrollos de la OIT que ayuden a entender la proyección que la noción de sector informal alcanzó en dicha institución.

Después de la Segunda Guerra Mundial y tras los esfuerzos de reconstrucción de Europa y Japón, varias instituciones comienzan a pensar en una estrategia similar para los países del Tercer Mundo. El bienestar material aparecía como un ideal de proyección universal, y la forma en que la OIT podía ayudar a alcanzarlo era colaborando en la gestión del proceso de transición económica que iba de una situación definida como tradicional a otra "moderna" donde ese bienestar podía ser garantizado. En el contexto de la Guerra Fría, y dado el compromiso resultado de la presencia de países de diversas orientaciones en la OIT, lo fundamental para gerenciar esa transición era una planificación del desarrollo, ya sea dentro de un modelo socialista, capitalista o "no alineado".

En todos los casos, el objetivo central era la formación de capital y la promoción de exportaciones, ya que la cuestión del empleo se resolvería con el crecimiento de ambos elementos. El mercado de trabajo encontraría un equilibrio, con una demanda en crecimiento que utilizase de forma eficiente la mano 
de obra, y con una oferta de empleados calificados, por eso los planes de la sección de Gerencia de Desarrollo - Management Development Branch -, y los planes de la sección de Formación Vocacional - Vocational Training Branch - respectivamente. ${ }^{12}$ Junto a estas dos secciones, la encargada de la Planificación de Mano de Obra - Manpower Planning Branch - era la tercera columna del Departamento para el Desarrollo de Recursos Humanos. Es aquí donde uno de los problemas centrales de esa versión del desarrollo comenzó a revelarse de forma sistemática: los puestos de trabajo (modernos) creados no alcanzaban a cubrir a la totalidad de la población. El modelo se escudaba en la variable temporal: esa desproporción iría siendo superada a medida que las sociedades atravesasen los diferentes estadios de desarrollo. ${ }^{\text {13 }}$

Ese desfasaje, consecuencia de la transición postulada entre los diferentes estadios de crecimiento, empezó a revelarse como algo más que temporario. Aun incorporando las previsiones más favorables de inversiones y crecimiento, la transición no iría a suceder de forma automática. Es entonces que el énfasis cambia y, en I969, la OIT lanza el Programa Mundial de Empleo - World Employment Programme (WEP) -, que tiene por objetivo colocar la generación de empleo en el centro de la planificación nacional y de los esfuerzos de desarro1lo. ${ }^{14}$ Con esa mudanza de énfasis suceden también algunos cambios institucionales y operacionales. La oficina que se ocupaba de la planificación de la mano de obra sale del Departamento de Desarrollo de Recursos Humanos y gana estatuto de Departamento dentro de la OIT, con el nombre de Departamento de Planificación y Promoción de Empleo.

Tres ramas componen dicho departamento: investigación, proyectos sectoriales y misiones de empleo. Estas misiones fueron una innovación para la época y una de ellas jugará un papel fundamental en la formulación del sector informal. Estaban centradas en análisis y diagnósticos en vez de restringirse a la asistencia técnica - forma hasta entonces prevaleciente. Eran equipos multidisciplinarios con especialistas de diversas instituciones y de diversos orígenes, trabajando dos o más meses en cada lugar.

La primera misión comprehensiva de empleo fue la de Kenia en I972, coordinada por Hans Singer y Richard Jolly. En el informe Employment, incomes and equity: a strategy for increasing productive employment in Kenya, publicado análisis de la situación en dicho país. Tal como Keith Hart, el informe de Kenia cuestiona el punto de partida asumido para pensar el problema del empleo; esto es, comenzar los análisis de empleo a partir de aquello que es registrado como tal. Como aparece en la introducción, "El problema con el empleo es que las estadísticas están incompletas omitiéndose un espectro de asalariados y personas autoempleadas, tanto hombres como mujeres, en aquello que llamamos 'sector informal'" (ILO, I972a: 5). Sector que, lejos de ser marginalmente productivo, es económicamente eficiente y generador de ganancias, a pesar de ser pequeño en escala y estar limitado por tecnología simple, por la escasez de capital y por la 
falta de relaciones con el otro sector de la economía ("formal” aparece entre paréntesis en el original).

Pero ¿qué son esas actividades? Veamos la cita del informe que se tornaría parámetro estándar de definición del sector informal:

Las actividades informales no están confinadas al empleo en la periferia de las ciudades principales, a determinadas ocupaciones o inclusive a actividades económicas. Por el contrario, las actividades informales son una manera de hacer las cosas, caracterizada por (a) facilidad de entrada, (b) dependencia de recursos indígenas, (c) propiedad familiar de las empresas, (d) pequeña escala de operación, (e) trabajo intensivo y tecnología adaptada, (f) destrezas adquiridas fuera del sistema escolar formal, y (g) mercados desregulados y competitivos. (ILO, I972a: 6).

El informe de Kenia pone el acento en la forma determinada en que las actividades son realizadas. Resalta de manera positiva el sector informal, rescatando la eficiencia, el carácter innovador y la resistencia del mismo. Destaca la falta de reconocimiento gubernamental - a veces su clara hostilidad hacia el sector -, y coloca la necesidad de incorporar dicho sector dentro del cuadro y de cualquier estrategia de desarrollo, en particular aquella que aparece formulada en el informe y que después se haría famosa en la discusión sobre desarrollo: la "redistribución con crecimiento".

\section{LAS VUELTAS DE LAS IDEAS}

A diferencia de otros conceptos utilizados en la descripción y gerenciamiento de lo social, la precisión con que es narrada la historia de la informalidad, en términos de autoría y momento de surgimiento, es algo que siempre me llamó la atención. Las decenas de artículos que trazan su historia y definición vuelven siempre sobre Keith Hart y la OIT en su misión de Kenia a principio de los I970. Sin embargo, al cotejar esas dos trayectorias aparece una cierta inconsistencia, que puede ser una cuestión anecdótica, pero tal vez nos ayude a entender la forma en que determinadas historias fueron contadas y los silencios que esas narrativas continúan produciendo.

La versión estándar es la que conté. Hart presenta el concepto en una conferencia en I97 I; en el I972, la misión de empleo de la OIT a Kenia lo toma y populariza y, en I973, Hart publica en forma de artículo el paper de la conferencia de I97I. Lo que no se cuenta es que en el informe de la misión de Kenia, en una nota al pie de página, la idea del sector informal es presentada como habiendo surgido del trabajo y staff del Institute of Development Studies de la Universidad de Nairobi. Si es así, ¿dónde entra Hart?

La conferencia Urban Unemployment in Africa fue realizada en el Instituto de Estudios del Desarrollo de la Universidad de Sussex, organizada por Richard Jolly y Rita Cruise O'Brien. ${ }^{15}$ Como vimos anteriormente, Richard Jolly coordinará junto a Hans Singer la misión de empleo de la OIT en Kenia; siendo figuras fundamentales en la historia de la economía del desarrollo. 
Uno de los participantes de la conferencia, el economista John Weeks, presenta el trabajo "The problem of urban imbalance", donde analiza la desigual distribución de empleo y oportunidades en las ciudades africanas. Para dar cuenta de todos aquellos que no tienen una relación salarial, pero que trabajan y obtienen ingresos, Weeks utiliza la expresión "the unnumerated sector". Según Keith Hart, en comunicación personal, Weeks encontró la idea de oportunidades informales de generación de ingresos presentada por él como muy útil - más apropiada que "sector no-enumerado" - y lo felicitó por el trabajo, así como lo hicieron dos participantes del Instituto de Estudios del Desarrollo de Nairobi. John Weeks va a formar parte del equipo convocado por Singer y Jolly para realizar la misión a Kenia y, según Hart, es él quien se sorprende al ver en el informe final la utilización del concepto de sector informal sin darle ningún crédito y mencionando a los investigadores de Nairobi como los creadores de la idea. Sorpresa que hará pública en una pequeña nota al respecto.

A lo anterior se sumará la cancelación del libro planeado por los organizadores con los papers presentados en la conferencia, por lo que el paper de Hart quedaría relegado en los archivos de las conferencias. El director del Journal of Modern African Studies, al tomar conocimiento de la historia, le propone a Hart publicar enseguida su artículo. Según Hart, todo este pequeño escándalo consiguió que muchas personas que comienzan a utilizar el concepto se encarguen de repetir que fue él quien acuño el término, algo que de otra manera no hubiera sucedido. ${ }^{16}$

Sólo para agregar otra voz en este relato, en la memorial lecture proferida por Richard Jolly en homenaje a Hans Singer, al comentar cuáles fueron los tres elementos que garantizaron el amplio impacto del informe sobre empleo de la misión de Kenia que coordinaran de forma conjunta, Jolly señala que uno de ellos fue la utilización del concepto de informalidad. Esto sucede meses después que Keith Hart lo reportara en su trabajo sobre Ghana, aclarando en una nota al pie que el mismo fue presentado en la conferencia organizada por él y Rita Cruise O’Bien, en septiembre de I97 I (Jolly, 2006: 5-6). Aquello que no había sido mencionado en el informe de 1972, aparece reconocido por uno de los directores de la misión al homenajear a su colega.

Además de este "efecto de autoría" - el posterior reconocimiento de los autores del concepto -, algo más importante sucede a raíz de estas idas y vueltas: la modificación de la naturaleza del concepto. Tal como Hart lo describiría:

La ironía de todo esto es que el informe de la OIT fue responsable por el hecho de acuñar un concepto que nunca fue mi intención de nombrar como tal. Yo simplemente hablé de oportunidades formales e informales de ingresos e inserté el término "sector informal" en la versión publicada de mi paper como resultado del uso del informe de la OIT con el cual yo estaba bien familiarizado por entonces. ${ }^{17}$ 
Claro que, aun utilizando la noción de sector informal, el paper de Hart no está construido desde una lógica que reifica al sector informal. Son varios los autores que van a señalar las diferencias entre la forma en que Hart utiliza el concepto y la forma que asume en el informe de la OIT. ${ }^{18}$ De acuerdo con Lisa Peattie, quien a mitad de los 1980 realiza una revisión del concepto de informalidad, en Hart sirve para dar cuenta de una variedad de actividades en la economía de los pobres urbanos, y aparece junto a otras categorías de la economía política en donde adquiere sentido. Por su lado, dice Peattie (I987: 854), "Cuando la OIT adopta el término, se transforma en una forma de describir la estructura de la economía como un todo en la terminología de los planificadores económicos". La introducción de la figura de "sector" trae una rigidez que inscribe a la idea de informalidad en la genealogía de los dualismos que influenciaron el pensamiento económico, no como herramientas conceptuales, sino como categorías de descripción de lo real, tal como antes funcionaba la dicotomía entre Moderno/Tradicional.

Es tal vez por esto que la teoría de la racionalización de Weber, que Hart reivindica como la inspiradora de la distinción entre formal e informal, quedó opacada en la utilización del concepto. Esta genealogía weberiana del concepto de informalidad, que Hart explorará posteriormente (I987 y 2006), subraya el crecimiento de la organización burocrática y el cálculo de ganancias y riesgos en las instituciones económicas occidentales; i.e. la emergencia del Estado racional-legal como garantía y soporte de espacios del universo social que adquieren un creciente grado de formalización. ${ }^{19}$ Es frente a esta dinámica que lo informal aparece iluminando todo aquel universo que queda fuera de visión a la luz de esa formalización.

Si la inscripción dentro del pensamiento dualista es una de las cuestiones que van a quedar adheridas al concepto como resultado de sus condiciones de surgimiento, la otra cuestión que merece ser destacada es aquella que se revela en la frase utilizada por John Weeks como "sector no-enumerado", pues precisamente muestra el interés por categorizar aquello que se escapaba a los números y que, como veremos, se transformará en objeto de cuantificación. Claro que la noción de informalidad se superpone con otras nociones utilizadas para describir lo que aparece fuera de las cuentas oficiales, retratado bajo el concepto de informalidad. Por ejemplo, la noción de underground economy desarrollada por el economista Vito Tanzi (I982), ${ }^{20}$ traducida como economía subterránea o como economía clandestina por diferentes investigadores. Algunos autores van a utilizar la expresión subterranean economy (Gutmann, I977). Otras conceptualizaciones muy comunes son black economy (mercado negro) y hidden economy (economía oculta). Un economista reconocido por sus propuestas para medir el fenómeno, Edgar L. Feige, utilizó a lo largo de su carrera las nociones de economía irregular (Feige, I979), sector no-observado (Feige, I98I) y economía subterránea e informal (Feige, I990). En esa búsqueda, la categoría de in- 
formal consiguió formalizarse de un modo tal que conceptos alternativos no lograron. Y, como muchas otras historias de formalización, la del concepto de informalidad está asociada a las trayectorias institucionales por las que fue desarrollándose.

\section{FORMATEANDO LO INFORMAL}

Una vez desembarcado en la OIT, la suerte del concepto de sector informal no fue nada lineal. El área de investigaciones sobre pobreza urbana - una de las líneas de investigación del Programa Mundial de Empleo - será el espacio donde el concepto se afiance y crezca. Años más tarde se constituirá en un programa de investigaciones en sí mismo, el "programa de investigaciones en el sector informal urbano". Harold Lubell, quien ingresa en I97 I, y S.V. Sethuraman, quien lo hace en I973, van a ser dos figuras fundamentales en la apropiación y difusión del concepto. Si bien no gana autonomía como tema principal de investigación, durante los años 1970 el "sector urbano informal" reemplazó el foco de los estudios sobre "desempleo urbano". ${ }^{21}$ Sin embargo, el concepto no es completamente asumido por la OIT, ni siquiera por equipos de investigación o misiones que lidiaban con "temas" informales. ${ }^{22}$

En la década del I980, la categoría de sector urbano informal se difunde, transformándose en uno de los cinco temas globales delineados por la OIT (plan 82/87), y entra a formar parte de la agenda de otros departamentos. Al final de la década es uno de los temas interdepartamentales prioritarios. Sin embargo, las prácticas institucionales habían cambiado: el énfasis se volvió al abordaje de acciones de intervención en contraposición con el carácter de diagnóstico y recomendación de las misiones.

Concebidas como remedios, las intervenciones difícilmente funcionaron con el sector informal. Las características de funcionamiento de la OIT son fundamentales para entender esas dinámicas. Por un lado, la manera en que se realizan la programación y el presupuesto reproduce formas de hacer independientemente de los resultados. ${ }^{23}$ Por otro lado, en el patrón de representación característico de la OIT - trabajadores, empleadores y gobierno -, el sector informal no tuvo un "defensor natural" en ninguna de las partes (Bangasser, 2000: I5).

De acuerdo con Bangasser, el grupo de los trabajadores se opuso consistentemente al trabajo sobre el sector informal desde los I970 hasta I984, año en que es aprobada la primera resolución llamando a los sindicatos a tomar un papel más activo en relación al sector informal. Los empleadores, aunque hoy suscriben una visión más positiva sobre el sector informal, continúan cuestionando su competencia desleal. La OIT desarrolló un trabajo que, si bien no desafió a esos sectores, tampoco aceptó su negativa a abordar el sector informal. Sin embargo, no fue un logro institucional (como el trabajo sobre género) sino por la perseverancia y perspicacia de algunos profesionales (Lubell y Sethuraman, Victor Tokman, George Niham y Carlos Maldonado). 
En la historia institucional del concepto de informalidad en la OIT, Paul Bangasser (2000: I6) caracteriza de forma crítica el abordaje centrado en intervenciones que remediasen la situación de los informales:

al concentrar su atención en 'ayudar' a aquellos que sufren de la informalidad (esto es, al concentrar en remediar los síntomas en vez de corregir las causas del sector informal), hemos conseguido reivindicar por tres décadas que estábamos respondiendo a esa enfermedad social virulenta sin tener que cambiar nuestro propio modus operandi ni arriesgar transformar en obsoletas las capacidades técnicas desarrolladas a lo largo de los años por la oficina.

Independientemente de las propuestas desarrolladas para el sector informal, ${ }^{24}$ el trabajo de la OIT sobre dicho sector asumió otra dimensión en la década del I990, cuando consigue incorporarlo en la discusión general de la organización y tornarlo una 'realidad' a ser registrada de forma sistemática. Incluida como aspecto principal en la agenda de la Conferencia Internacional de Trabajo en I99I, la discusión sobre el sector informal es presentada en el reporte elaborado por el director general, The dilemma of the informal sector (Report of the Director General, International Labour Conference, 78th Session, I99I). Por primera vez, empleadores, trabajadores y gobernantes tuvieron que exponer sus posiciones sobre el tema, dejando así, de ser una discusión exclusivamente de especialistas. La respuesta del director a las diferentes posiciones relevadas subraya tres cuestiones que van a orientar las acciones de la institución: la aplicación igualitaria de estándares fundamentales de derechos humanos para todos, la utilización de reglas y leyes simples como prerrequisitos para la legalización gradual del sector informal, y la necesidad de acondicionar la ley sin hacerla retroceder en términos de garantías.

Los esfuerzos por sistematizar las formas de registro del sector informal se cristalizan en las discusiones de aquellos que tornan numéricamente reales las abstracciones de los conceptos. En I982, en la I $3^{\text {a }}$ Conferencia Internacional de Estadísticos del Trabajo (en adelante utilizaré su sigla en inglés, ICLS), se había sugerido que cada país desarrollase metodologías y programas para la recolección de estadísticas sobre el sector informal. En I987, durante la I $4^{\underline{a}}$ ICLS, la resolución de la conferencia informa que sería desarrollado un marco conceptual que delineará herramientas para registrar sistemáticamente las "formas no estandarizadas de empleo e ingresos". Esta resolución colocó al sector informal en la agenda de la I $5^{\underline{a}}$ ICLS que tuvo lugar en enero de I993, lo que exigió la realización de un reporte para dicha conferencia. ${ }^{25}$

Paralelamente, se estaba haciendo una nueva versión del System of $\mathrm{Na}$ tional Accounts (SNA); marco conceptual utilizado por los sistemas estadísticos financieros y económicos para calcular el producto bruto de cada país y otras figuras comparativas. Después de una década de trabajo, la revisión del SNA en 1993 bajo la dirección de la comisión estadística de la ONU, con la participación de Eurostat, el FMI, el Banco Mundial, la OECD y varias otras agencias, 
incorporó la distinción de los sectores formal e informal. Reconociendo el papel de la OIT como experta en el asunto, el SNA incluyó como anexo la resolución de la I $5^{\underline{a}}$ Conferencia Internacional de Estadísticos del Trabajo (ICLS) realizada meses antes, la cual fue adoptada formalmente y recomendada por el Consejo Económico y Social de la ONU.

Desde I993, transformado en algo mensurable y oficializado internacionalmente como parte del universo que cada país debe observar y registrar, el sector informal pasó a tener una realidad que será regularmente confirmada en cada censo y en cada lugar. Claro que para eso debemos tener las herramientas adecuadas, algo cuya definición incluye discusiones y cuestionamientos sobre el contenido de dicho sector. Habiendo alcanzado un estatus real, el trabajo en los últimos años consistió en calibrarlo, tal como puede ser interpretada la creación en I 997 del Grupo de Expertos en Estadísticas del Sector Informal, el Grupo Delhi, con el objetivo de preparar el manual para mediciones del sector informal, el cual es finalmente presentado en $20{ }_{3}$ (ILO, 2013).

\section{REFLEXIONES DESDE AMÉRICA LATINA}

La difusión del concepto de informalidad en América Latina en los formatos hasta ahora descriptos estuvo vinculada a la actividad del Programa de Recuperación de Empleo en América Latina (PREALC), el cual expresa la posición de la OIT en la región. Un análisis pormenorizado de los desarrollos de la categoría en estos lados del mundo significaría otro trabajo, especialmente porque es uno de los espacios donde ingresa en debates que van a tener un profundo impacto en la discusión internacional. No solo a partir de América Latina, sino del continente americano en general. Los trabajos de Victor Tokman y Vanesa Cartaza, son fundamentales para entender la articulación de las discusiones internacionales con el contexto americano; de gran importancia son los trabajos de Alejandro Portes, Robert Castells y Saskia Sassen que reorientaran la mirada sobre la informalidad como fenómeno característico de los países subdesarrollados, incorporando a la discusión los procesos de reestructuración económica en países desarrollados. También el trabajo de Hernando de Soto sobre la informalidad como respuesta popular al mercantilismo rígido de es tado y la idea que se tornaría clave posteriormente a propósito de los costos de la legalidad.

La historia singular del concepto de informalidad, que no es otra cosa que la narrativa que tenemos de ella y que es reproducida recurrentemente en artículos, libros, tesis y seminarios, presenta una singularidad especial al ser comparada con otros conceptos utilizados en el análisis social: una precisión particular sobre el momento y los actores vinculados a su formulación. Si a la luz del material hasta aquí presentado podemos concluir que esa precisión está vinculada a ciertas disputas o afirmaciones autorales sobre el concepto, lo cierto es que las mismas tuvieron un efecto canónico sobre el relato que 
seguimos contándonos. No dar por sentado ese relato tal vez nos permita recuperar otras genealogías a partir de las cuales formular nuevas preguntas.

Una formulación paralela y convergente con la de Keith Hart la encontramos en la tesis de maestría de Luiz Antonio Machado da Silva (I97 I), Mercados metropolitanos de trabalho manual e marginalidade, con fecha de mayo de I97 I y defendida en el Programa de Postgrado en Antropología Social del Museo Nacional (Universidad Federal de Rio de Janeiro) bajo la dirección de Roger B. Walter. En ella, Machado da Silva analiza el mercado de trabajo de las metrópolis brasileñas a la luz de los subsistemas denominados "mercado formal" y "mercado no-formalizado". La dicotomía formal/informal presentada por el autor, nos coloca en el mismo terreno sobre el que desarrolla sus ideas Keith Hart: la discusión sobre empleo y su relación con el marco jurídico-institucional de los universos sociales analizados. Y así, como en Hart, el abordaje etnográfico que permite presentar un universo diversificado de trabajos no-formalizados entre los sectores populares urbanos también ilumina, de acuerdo con Mariana Cavalcanti (2018: 80), como "lo formal y lo informal se conectan y combinan en lo cotidiano". 26

Muchas páginas podrían ser escritas a la luz de ese material, sin embargo, quiero concluir señalando la razón del ejercicio hasta aquí realizado. Por un lado, es fundamental considerar seriamente la historia institucional de las ideas para comprender los efectos que ellas tienen en los universos sociales sobre los que trabajamos. Efectos que van desde la implementación de políticas específicas, hasta la incorporación de determinadas ideas que influyen en las formas de construir la propia identidad de las personas. Por otro lado, las historias institucionales e institucionalizadas a partir de las trayectorias singulares de la vida social de las ideas no necesariamente son las únicas que podríamos contarnos. ${ }^{27}$ Indagar en esas otras historias tal vez nos permita superar algunos impases en que a veces nos colocan ciertos desarrollos conceptuales. En este sentido, ubicar la discusión sobre informalidad en el histórico de los debates sobre marginalidad, sobre las dicotomías urbano-rural, tradición-modernidad y otras formas en las cuales fueron discutidas las transformaciones del mundo contemporáneo, puede ser renovador.

Recibido I7/4/20I9| Aprobado 7/IO/20I9

Fernando Rabossi es profesor del Departamento de Antropología Cultural y del Programa de Posgrado en Sociología y Antropología de la Universidad Federal de Rio de Janeiro. Su foco de investigación es la relación entre reglas y prácticas económicas. Es autor del libro En las calles de Ciudad del Este: una etnografía del comercio de frontera (2008) y de varios artículos sobre economías y políticas informales, procesos de formalización, mercados, fronteras, circulación y etnografía. 


\section{NOTAS}

I Por ejemplo, el presidente de una de las asociaciones de vendedores de la calle (mesiteros, tal como son llamados), describiendo la asociación que presidía, señalaba: "Vos sabés que la asociación le sirve... especialmente para nosotros, como nosotros somos trabajadores informales, ¿verdad? No dependemos de ningún patrón, nosotros mismos es patrón, y... también es empleado, ¿verdad?" (Entrevista con Gregorio Villalba, 28.6.200I, Ciudad del Este).

2 La descripción del presidente de una asociación de cambistas después de haber sido reconocidos por el Banco Central del Paraguay para operar con cambio, era la siguiente: "los trabajadores cambistas de la calle, no solamente de acá sino de todo el país, dejó de ser un trabajador informal y entonces pasa a ser un trabajador formal, porque estamos tributando al Estado paraguayo" (Entrevista con Mario Sánchez, I5.5.200I, Ciudad del Este).

3 La primera versión de este trabajo fue presentada en el VIII Congresso Argentino de Antropologia Social, 2006 (Salta, Argentina) y retrabajada para el XXVII International LASA Congress, 2007 (Montreal, Canada). Muchas de las ideas han sido discutidas con los colegas del Núcleo de Pesquisas em Cultura e Economia (NUCEC), en particular en el curso Economias Populares e Formas de Governo, dictado en 2012 en el PPGSA y el PPGAS de la UFRJ, junto a Federico Neiburg, Eugenia Motta y Daniel Hirata. Desde 2013, con Daniel Hirata estamos trabajando las discusiones sobre informalidad a partir de los procesos de formalización y escribiendo la segunda parte de este artículo: Os caminhos das formalizações. La bibliografía sobre informalidad es inmensa y no es objetivo de este artículo hacer una revisión de ella. En el caso brasileño, merecen ser destacados los trabajos de Luis Antonio Machado da Silva - algunos de los cuales serán abordados en este texto -, Claudio Salvadori Dedecca, Maria Cristina Cacciamali Souza, Jacob Lima, y Roberto Véras de Oliveira y sus grupos de investigación. Alexandre Barbosa (20II) aborda vários elementos aquí desarrollados, insertos en el contexto más amplio de las discusiones en Brasil. Adalberto Cardoso (2016) aborda de una forma novedosa los procesos de formalización que estamos analizando com Daniel Hirata. 
4 La relación entre ideas económicas eruditas y ordinarias es uno de los ejes de investigación del Núcleo de Pesquisas em Cultura e Economia (NUCEC) del PPGAS/Museu Nacional y del PPGSA/IFCS de la Universidad Federal de Rio de Janeiro. Ver, por ejemplo, Pantaleón, 2004; Neiburg, 2006; Braum 2009; Motta, 20I0 y 20I9; Onto, 2014 y 20I6; Rabossi, 2018. El interés en este eje de investigación tiene diversas inspiraciones. En mi caso, deriva de la confluencia de los estudios sobre dispositivos y artes de gobierno (Foucault, 2006), sobre cuantificación (Desrosières, I993; Porter, I995) y, en particular, sobre la relación entre conocimiento económico y economía (Callon, I998; Mitchel, 2005).

5 El resto de las citas en idioma extranjero fueron traducidas por el autor.

6 En el Jornal of Modern African Studies, II/I, p. 6I-89. El texto original aún no ha sido traducido al portugués.

7 El caso de Atinga, un ex-soldado que por unos años mantiene un bar (Hart, I973: I55-I56).

8 Por ejemplo, propondrá un abordaje de input-output sobre el flujo de ingresos formales e informales para analizar el peso relativo de cada uno de ellos y el grado de preponderancia en distintos sectores sociales (Hart, I973: I59).

9 Después de distanciarse de la discusión sobre informalidad, el autor volverá a discutir el concepto en diversas ocasiones, recuperando el contexto de su formulación, las inspiraciones intelectuales que lo influenciaron y las transformaciones ocurridas en los últimos tiempos que han modificado la agenda de la discusión y el alcance del concepto. Ver Hart, I992, 2006 y 2015.

Io El relato que sigue está basado en el texto de Paul Bangasser (2000) sobre la historia institucional del concepto de informalidad en la OIT. Como miembro de dicha organización, el relato de Paul Bangasser es extremamente interesante, combinando un análisis institucional con su experiencia personal y la de sus colegas de trabajo. Para una visión comprensiva de la historia intelectual de las Naciones Unidas en general y de la OIT en particular, ver Ward, 2004; Emmerij, Jolly \& Weiss, 2005; Jolly, Emmerij \& Weiss, 2005; Jolly, 2006. 
I I La OIT emerge de los acuerdos internacionales posteriores a la Primera Guerra Mundial. En I9I9 se crea la constitución de la OIT, anexada al Tratado de Versalles como su Parte XIII. Fue formada con el objetivo de abordar las condiciones y los problemas del trabajo, a través de convenciones y sugestiones y de la estandarización de las condiciones de trabajo. Posee una estructura tripartita (patrones, trabajadores y gobernantes). Cada país - de los I85 que son miembros - está representado por dos miembros del gobierno, un miembro de las organizaciones de empleadores y un representante de las organizaciones de trabajadores. En 1946 fue incorporada dentro de la Organización de las Naciones Unidas, siendo su primera agencia especializada. Sobre la trayectoria de la OIT em Brasil, ver Barroso, 2017.

I2 Ambas secciones pertenecen al Departamento para el Desarrollo de Recursos Humanos, siendo las piezas principales en la cooperación ofrecida en aquel entonces por la OIT. Esos dos programas crecen enormemente con los recursos del recién creado PNUD.

I3 El clásico de esa formulación es el libro de Walt Rostow (I960) sobre las etapas del crecimiento económico.

I4 Paul Bangasser (2000: Io), miembro de la OIT, señala a propósito de las posiciones que estaban en disputa: "To be sure, this 'residual self-regulating labour market' thesis never sat well with much of the ILO community, both within the Office and among the constituents (especially on the workers group side). But it was a widely held view among respectable mainstream economists; and economists tended to dominate the development debate. The WEP was, in effect, a direct attack on this conventional economists' wisdom. The basic WEP counter thesis was that employment should be seen as a central component of development efforts, not as an eventual result of them. It should figure prominently both at the planning as well as the implementation stages and at the macro as well as the micro levels".

I5 Para una descripción de la conferencia, ver O’Brien, I972.

I6 Este relato está basado en un intercambio con Keith Hart a raíz de mi pregunta sobre la inconsistencia entre el reconocimiento público de su persona como autor del con- 
cepto y la indicación en el informe de Kenia de la OIT de que el concepto habría sido forjado por miembros del staff de la Universidad de Nairobi. Narrar el episodio desde nuestro dialogo me permitió iluminar una cuestión que fue tornándose para mi en una intriga: ¿por qué la autoría del concepto de informalidad aparece recurrentemente ratificada en la introducción al tema? Un trabajo más profundo, obviamente, necesitaría algunas entrevistas y un trabajo de archivo sistemático.

I7 Comunicación personal. I5 septiembre 2006.

I8 Ver por ejemplo Rempel \& House, I978; Peattie, I987.

I9 En "Market and State after the Cold War: the informal economy reconsidered", Hart (I992) interpreta el concepto de "economía informal" emergiendo en el África poscolonial durante los I96os para denotar un aspecto de la relación entre el Estado y el mercado. State capitalism capitalismo estatal - es el concepto clave utilizado por el autor para dar cuenta de la centralidad del Estado - y no del mercado - en la reproducción de las relaciones capitalistas de producción durante el siglo XX, producto de la convergencia de la burocracia estatal y el capitalismo industrial corporativo.

20 Para una re-evaluación de lo realizado en los 20 años posteriores a la presentación de The underground economy in United States and abroad, ver Tanzi, I999. Vito Tanzi fue por 30 años director del Departamento de Asuntos Fiscales del Fondo Monetario Internacional, siendo considerado uno de los creadores de los principios de la tributación moderna.

2 I Un estado de la cuestión a inicio de los I980 desde la óptica de la OIT, está en ILO, I984.

22 Tales como las misiones de Irán (ILO, I972b) y Filipinas (ILO, I974).

23 ¿Por qué la apuesta a los mismos remedios a pesar de la falta de resultados? Otra vez, las palabras de Paul Bangasser son iluminadoras. Según él, el funcionamiento y la estructura de la OIT nos permiten entender un poco el porqué de esa permanencia. Cada dos años hay que formular una programación y un presupuesto, aparentemente un proceso técnico orientado por las demandas de los 
miembros constituyentes. Cada oficina hace un pedido que, de hecho, son tres: uno con los gastos actuales, otro con más y otro con menos (esos pedidos son llamados de shopping lists). El contenido técnico es elaborado por el staff y por la estructura organizacional que ya estaba en base a la distribución de recursos existentes. Cuando los Estados miembros votan (seis meses antes que expire el actual presupuesto), hay poco margen de maniobra para concebir alternativas (Bangasser, 2000: I4-I5).

24 El Proyecto Interdepartamental I994-I995 de la OIT sobre el sector informal estuvo centrado en mejorar la productividad de las actividades de ese sector, luchar por la protección social adecuada a los estándares internacionales para trabajadores y productores, y promover y reforzar sus organizaciones e instituciones. Para ello, realizaron cursos de entrenamiento y seminarios en tres ciudades: Bogotá, Dar es Salaam y Manila.

25 En dicho reporte se establece la unidad económica (empresa) como medida más apropiada para definir al sector informal (no el trabajador).

26 La tesis de maestria de Luiz Antonio Machado da Silva fue recientemente publicada en el libro 0 mundo popular: trabalho e condições de vida, que reúne los trabajos de sociología económica del autor, organizado por Mariana Cavalcanti, Eugênia Motta e Marcella Araujo (Machado da Silva, 2018).

27 Para seguir la discusión, deberíamos analizar el desembarque del concepto de informalidad en el Banco Mundial en los años I990 y la importancia que adquirió en la última década en dicha institución. Desembarque y expansión paralelos al cambio de referente normativo que pauta las discusiones sobre informalidad y los procesos de formalización: del trabajo a la empresa. Junto con Daniel Hirata, desarrollamos una primera aproximación y descripción de esos referentes en el texto "Comércio ambulante no Rio de Janeiro: entre formalizações e a informalidade" (Hirata \& Rabossi, 2017). 


\section{REFERENCIAS BIBLIOGRÁFICAS}

Bangasser, Paul E. (2000). The ILO and the informal sector: an institutional history. Employment Paper, 9.

Barbosa, Alexandre de Freitas. (20II). O conceito de trabalho informal, sua evolução histórica e o potencial analítico atual: para não jogar a criança fora junto com a água do banho. In: Oliveira, Roberto Véras de; Gomes, Darcilene \& Targino, Ivan (orgs.). Marchas e contramarchas da informalidade do trabalho: das origens às novas abordagens. João Pessoa: Editora da UFPB, p. I05-I59.

Barroso, Márcia Regina Castro (2017). A OIT e o mundo do trabalho no Brasil: trajetória e ações institucionais em períodos democráticos. Tese de Doutorado. PPGSA/Universidade Federal do Rio de Janeiro.

Braum, Pedro. (2009). O estado da ciência e a ciência do Estado: A FGV e a configuração do campo das ciências econômicas no Brasil. Dissertação de Mestrado. PPGAS/Universidade Federal do Rio de Janeiro.

Callon, Michel. (I998). Introduction: The embeddedness of economic markets in economics. In: Callon, Michel (org.). The laws of the markets. Oxford: Blackwell Publishers, p. I-57.

Cardoso, Adalberto. (20I6). Informality and public policies to overcome it. The case of Brazil. Sociologia \& Antropologia, 6/2, p. 32I-349.

Cavalcanti, Mariana. (2018). O cotidiano do trabalho na cidade. In: Machado da Silva, Luiz Antonio. O mundo popular: trabalho e condições de vida. Rio de Janeiro: Papéis Selvagens, p. 77-82.

Desrosières, Alain. (1993). La politique des grandes nombres. Paris: Éditions Découverte.

Emmerij, Louis; Jolly, Richard \& Weiss, Thomas G. (2005). Economic and social thinking at the UN in historical perspective. Development and Change, 36/2, p. 2II-235.

Feige, Edgar L. (I990). Defining and estimating underground and informal economies: the new institutional economics approach. World Development, I8/7, p. 989-1002. Feige, Edgar L. (I98I). The UK's unobserved economy: a preliminary assessment. Economic Affairs, I/4, p. 205-2I2. 
Feige, Edgar L. (I979). How big is the irregular economy? Challenge, I2, p. 5-13.

Foucault, Michel. (2006) [1978]. Seguridad, territorio, población: Curso en el College de France: 1977-1978. Buenos Aires: Fondo de Cultura Económica.

Gutmann, Peter. (1977). The subterranean economy. Financial Analyst Journal, 35, p. 26-28.

Hart, Keith. (20I5). How the informal economy took over the world. In: Mörtenböck, Peter et al. (eds.). Informal market worlds reader. Amsterdam: NAIoio Publishers, p. 33-44. Hart, Keith. (2006). IE + IT = ED? Is informal economy plus informational technology a path towards economic democracy? Workshop Clusters, Network Organization and the Informal Economy (Serie Rethinking Economies), Bologna.

Hart, Keith. (1992). Market and State after the Cold War: the informal economy reconsidered. In: Roy Dilley (ed.). Contesting markets: analyses or ideology, discourse and practice. Edinburg: Edinburg University Press, p. 214-227.

Hart, Keith. (1987). Informal economy. The New Palgrave - A Dictionary of Economic Theory and Doctrine, v. 2.

Hart, Keith. (I973). Informal income opportunities and urban employment in Ghana. Journal of Modern African Studies, II/I, p. 6I-89.

Hirata, Daniel \& Rabossi, Fernando. (20I7). Comércio ambulante no Rio de Janeiro: entre formalizações e a informalidade. Estudo Estratégico, I3.

ILO. (2013). Measuring informality: a statistical manual on the informal sector and informal employment. Geneva: ILO Publications.

ILO. (I984). Urbanisation, informal sector and employment: a progress report on research, advisory services and technical cooperation. Geneva: ILO World Employment Programme. ILO. (I974). Sharing in development: a programme of employment, equity and growth for the Philippines. Geneva: ILO Publications.

ILO. (I972a). Employment, incomes and equity: a strategy for increasing productive employment in Kenya. Geneva: ILO Publications. 
ILO. (I972b). Employment and income policies for Iran. Geneva: ILO Publications.

Jolly, Richard. (2006). Hans Singer: the gentle giant. Memorial lecture presented in Geneva, International Labor Organization.

Jolly, Richard; Emmerij, Louis \& Weiss, Thoms G. (2005). The power of UN ideas: lessons from the first 60 years. New York: United Nations Intellectual History Project.

Machado da Silva, Luiz Antonio. (2018). O mundo popular: trabalho e condições de vida. Org. Mariana Cavalcanti, Eugênia Motta \& Marcella Araujo. Rio de Janeiro: Papéis Selvagens.

Machado da Silva, Luiz Antonio. (I97I). Mercados metropolitanos de trabalho manual e marginalidade. Dissertação de Mestrado. PPGAS/Universidad Federal de Rio de Janeiro.

Mitchell, Timothy. (2005). The work of economics: how a discipline makes its world. European Journal of Sociology, 46, p. 297-320.

Motta, Eugênia. (20I9). Resistência aos números: a favela como realidade (in)quantificável. Mana, 25/I, p. 72-94.

Motta, Eugênia. (20Io). Trajetórias e transformações no mundo da economia solidária. Tese de Doutorado. PPGAS/Universidade Federal do Rio de Janeiro.

Motta, Eugênia et al. (20I4). Foreword - Ethnographies of Economy/ics: Making and Reading. Vibrant - Virtual Brazilian Anthropology, I I/I, p. 50-55.

Neiburg, Guillermo F. (2006). Inflation: economists and economic cultures in Brazil and Argentina. Comparative Study of Society and History, 48/3, p. 604-633.

O'Brien, Rita Cruise. (1972). Urban unemployment in Africa. The Journal of Modern African Studies, Io/I, p. Iog-II2.

Onto, Gustavo Gomes. (2016). O mercado como um contexto: delimitando o problema concorrencial de uma aquisição empresarial. Horizontes Antropológicos, 22, p. I55-I84. Onto, Gustavo Gomes. (20I4). The market as lived experience: on the knowledge of markets in antitrust analysis. Vibrant, Florianópolis, II, p. I59-I90.

Pantaleón, Jorge F. (2004). Uma nação sob medida: Estatísticas, economia e planificação na Argentina (I9I8-I952). Tese 
de Doutorado. PPGAS/Universidade Federal do Rio de Janeiro.

Peattie, Lisa. (1987). An idea in good currency and how it grew: the informal sector. World Development, I5/7, p. 85 I860.

Porter, Theodore. (1995). Trust in numbers: the pursuit of objectivity in science and public life. Princeton: Princeton University Press.

Rabossi, Fernando. (2018). Reaching the "Bottom of the pyramid": entrepreneurial strategies at the margins of Brazil. Cartografie Sociali: Rivista di Sociologia e Scienze Umane, 6, p. 57-72.

Rabossi, Fernando. (2004). Nas ruas de Ciudad del Este: vidas e vendas num mercado de fronteira. Tese de Doutorado. PPGAS/Universidade Federal de Rio de Janeiro.

Rempel, Henry \& House, William J. (I978). The Kenya employment problem: an analysis of the modern sector labour market. Nairobi: Oxford University Press.

Rostow, Walt Withman. (I960). The stages of economic growth: a non-communist manifesto. Cambridge: Cambridge University Press,

Tanzi, Vito. (I999). Uses and abuses of estimates of the underground economy. The Economic Journal, Io9, p. 338347.

Tanzi, Vito (ed.). (1982). The underground economy in United States and abroad. Lexington, Mass.: Lexington Book.

Ward, Michael. (2004). Quantifying the world: UN ideas and statistics. Bloomington/Indianapolis: Indiana University Press. 


\section{OS CAMINHOS DA INFORMALIDADE}

Resumo

Desde a década de I970, uma das chaves de análise das economias dos chamados países em desenvolvimento tem sido o conceito de informalidade. A adoção desse conceito por organismos internacionais e sua utilização para dar conta dos processos econômicos e sociais desses países é um campo frutífero para observar os processos de conceitualização, medição e construção de determinados fenômenos/objetos como focos de políticas. Surgido no campo da antropologia e do desenvolvimento, a vida social do conceito de informalidade está vinculada a sua circulação por diversas disciplinas. $\mathrm{O}$ trabalho proposto apresenta um balanço do desenvolvimento do conceito de informalidade, seu contexto de surgimento, sua incorporação a agendas de diversas agências internacionais e as tentativas atuais de novas formulações que deem conta do campo anteriormente retratado pelo conceito. O caso da informalidade permite refletir sobre a relação entre diversas linguagens disciplinares e a economia como disciplina, bem como sobre a ampliação dessas linguagens para abordar diversos aspectos do mundo contemporâneo.

\section{THE PATHS TO INFORMALITY}

Abstract

Since the I970s, one of the key concepts used to analyse the economies of so-called developing countries has been informality. The adoption of this concept by international organizations and its use to account for the economic and social processes of these countries is an ideal field in which to observe how certain phenomena are conceptualized, measured and constructed as focal policy issues. Emergent from the field of anthropology and development, the social life of the concept of informality is linked to its circulation through various disciplines. In this paper, I present the concept of informality, the context of its emergence, its incorporation into the agendas of various international agencies, and the current attempts to re-formulate it. The case of informality presents interesting aspects to reflect on the relationship between various disciplinary languages and economics as a discipline, as well as the expansion of these languages to address various facets of the contemporary world.
Palavras-chave

Informalidad;

OIT;

historia conceptual;

trayectoria institucional.

\section{Keywords}

Informality;

ILO;

conceptual history;

institutional trajectory. 